\title{
LOS SISTEMAS DE PARTIDOS EN ITALIA: UNA EVOLUCIÓN INACABADA'
}

\section{Party systems in Italy: An on-going evolution}

\author{
GIANFRANCO PASQUINO \\ Johns Hopkins University \\ gpasquino@johnshopkins.it
}

Cómo citar/Citation

Pasquino, G. (2020).

Los sistemas de partidos en Italia: una evolución inacabada.

Revista de Estudios Políticos, 189, 19-39.

doi: https://doi.org/10.18042/cepc/rep.189.01

\section{Resumen}

En la segunda posguerra Italia ha tenido tres sistemas de partidos. Siguiendo la lección de Sartori se impone «contar los partidos que cuentan». El primer sistema (1946-1992) fue definido como pluralismo polarizado debido a la presencia de dos partidos, el PCI y el MSI, diversamente antisistema. El segundo sistema (1994-2013) fue simplemente un sistema multipartidista, nunca estabilizado, caracterizado por un tipo de competición bipolar. Después de 2013 el sistema ha continuado siendo multipartidista, pero la competición es multipolar. Levemente antisistema, el M5S se ha convertido en partido de gobierno, a la vez que todos los demás partidos existentes también continúan transformándose en un contexto marcado por la alta volatilidad electoral. Como resultado de este cuadro, no es posible afirmar que el sistema de partidos se haya consolidado. Al contrario, debe ser definido como no estructurado.

\section{Palabras clave}

Partidos; sistema de partidos; polarizacion; escisión; volatilidad; deconstruccion.

$\overline{1}$ Texto traducido por Jorge del Palacio. 


\section{Abstract}

In the second post-war period, Italy has so far had three party systems. Following Sartori's teachings, one must "count the parties that count». The first party system, 1946-1992, has to be defined polarized pluralism, including two differently antisystem parties, the PCI and the MSI. The second party system, 1994-2013, was simply a multiparty system, not stabilized, having an occasional bipolar competition. Since 2013 the system is multiparty, but the competition has become multipolar. Mildly anti-system, the 5 Star Movement has obtained governmental offices. All existing parties continue to evolve. Electoral volatility is quite high. It is impossible to maintain that the party system is consolidated. On the contrary, it deserves to be defined unstructured.

\section{Keywords}

Parties and party systems; polarization; deconstruction; cleavages; volatility. 


\section{SUMARIO}

I. INTRODUCCIÓN. II. ALGUNAS REFERENCIAS AL SISTEMA DE PARTIDOS $1946-$ 1992. III. HUNDIMIENTO, RECUPERACIÓN Y RECAÍDA: 1994-2008. IV. 2007: UN NUEVO PARTIDO, NO UN NUEVO SISTEMA DE PARTIDOS. V. LA DESESTRUCTURACIÓN DEL SISTEMA DE PARTIDOS. VI. BALANCE Y VALORACIÓN FINAL. BIBLIOGRAFIA.

\section{INTRODUCCIÓN}

Este artículo está dedicado al análisis de aquellos que considero los tres sistemas de partidos que Italia ha tenido desde 1946 hasta hoy, los cuales han sido eficazmente identificados y analizados por Valbruzzi (2013). Trataré, sobre todo, de clarificar qué definición de los sistemas de partidos me parece analíticamente útil. Pasaré a las características que permiten, de hecho imponen, diferenciar con precisión tres sistemas de partidos. Me concentraré, en particular, en la transición del segundo al tercer sistema de partidos. La conclusión, en definitiva, será problemática. Italia tiene un sistema de partidos no consolidado, solo en parte despolarizado, pero también desestructurado. Por tanto, expuesto a transformaciones, más o menos imprevisibles, que dependerán además, y no poco, de una enésima y no improbable reforma de la ley electoral. Sobre esto, obviamente, es difícil aventurar previsiones salvo una: servirá antes a los intereses particulares de los partidos que a ofrecer una buena representación política y parlamentaria a los ciudadanos-electores.

Tradicionalmente la imagen de Italia ha sido la de un país con un sistema político (y de un régimen democrático) al mismo tiempo inestable y que, sin embargo, no cambiaba jamás. Como todas las imágenes, esta contenía una buena dosis de correspondencia con la realidad. Limitándome tan solo al sistema de partidos, que es el objeto de este artículo, no hay duda de que entre 1946 y 1992, con la mutación que comienza con esta elección, el sistema permaneció igual y funcionó en sustancia sin variaciones dignas de hacer notar. En la definición original de Sartori, ya en 1966 y completamente en 1976, el sistema de partidos italiano era definido como "pluralismo polarizado» ${ }^{2}$. Resulta útil,

2 No fue nunca un «bipartidismo imperfecto», título de un exitoso libro de Giorgio Galli (1967): ni bipartidismo ni imperfecto (tampoco sabría qué ejemplo ofrecer de 
quizás indispensable, recordar los criterios sobre los cuales Sartori clasifica los sistemas de partidos porque también servirán para describir y analizar el sistema de partidos italiano del 2008 hasta hoy (y mañana). Para definir correctamente un sistema de partidos se necesita, sobre todo, contar los partidos. Pero se necesita contar los partidos que cuentan, es decir, aquellos cuyo poder incide sobre la formación de las coaliciones de Gobierno e influye sobre su funcionamiento. De un lado, algunos partidos tienen poder de coalición, incluso cuando no están en el Gobierno; de otro lado, algunos partidos, aunque es probable que no lleguen jamás al Gobierno, han ejercitado y ejercitan un poder de chantaje. A saber, son capaces de presionar desde fuera al Gobierno determinando la formación de la coalición, haciendo difícil y, en ocasiones, imposible tomar algunas decisiones y políticas públicas específicas, o incentivando alguna toma de posición como la formulación de políticas públicas diferenciadas. Tanto el poder de coalición como el poder de chantaje no dependen exclusivamente de los votos y de los escaños de los respectivos partidos, sino también, y mucho, de su posición en el continuum derecha-izquierda, como en su capacidad para dar representación a algunos intereses. En general, el análisis de un sistema de partidos se debe centrar en la modalidad de la interacción entre los partidos y, en la medida de lo posible, de las interacciones entre los partidos y el electorado (Eckstein, 1968).

\section{ALGUNAS REFERENCIAS AL SISTEMA DE PARTIDOS 1946-1992}

Un sistema de partidos que responde al modelo del pluralismo polarizado está constituido, en primer lugar, por un número de partidos que Sartori sugiere que será, probablemente, superior a seis. Casi de manera inevitable, la coalición de Gobierno estará formada por tres o más partidos. Esta circunstancia implicará negociaciones delicadas, complejas y en ocasiones largas para la formación del Gobierno. También, de manera inevitable, implicará conflictos y tensiones en la propia actividad de gobierno. Resulta muy probable que los partidos en el Gobierno no sean lo suficientemente homogéneos, que muestren prioridades diferentes, que tiendan a poner de manifiesto su propia especificidad, que pretendan presentarse y posicionarse para hacerse visibles y fácilmente identificables para su electorado de referencia, que traten de atribuir a los demás la culpa por lo mal hecho o no realizado y que no acepten

«bipartidismo perfecto»). Naturalmente, los partidos italianos fueron siempre más de dos, entre siete y nueve, como fueron más de dos los partidos que contaron políticamente para formación de las coaliciones de Gobierno y el funcionamiento integral del sistema. 
responsabilidades, cargando el muerto a otros: buckpassing. En aquello que atañe al pluralismo, el resultado de la coalición será probablemente positivo. $\mathrm{Al}$ menos en cuanto al número de partidos y su capacidad para representar distintas preferencias e intereses sociales, políticos y económicos. Sin embargo, el número de partidos tendrá efectos potencialmente negativos sobre la cohesión y la duración de la coalición de Gobierno.

¿Cuál es el significado del adjetivo polarizado utilizado por Sartori? El número de partidos, su pluralismo, se refiere a la forma del sistema. La polarización se refiere específicamente al funcionamiento, a la dinámica que preside el sistema. Un sistema de partidos es polarizado cuando los polos resultan ser más de dos o cuando entre los dos polos se interpone una distancia ideológica notable, al punto de convertir en prácticamente imposible una coalición con y entre ellos. Obviamente, tres o más polos hacen imposible, tanto por definición como políticamente, una competición bipolar. Además, si hay dos polos, a la izquierda y la derecha del polo que se ubica en el centro, y sobre el cual descansa el sistema de manera inevitable, la competición no se dará en el centro, sino contra el centro. No será centrípeta. Aún más, en el esfuerzo por arrebatar electores al centro se caracterizará como centrifuga. Los partidos que se posicionan en los polos de la izquierda y la derecha estarán siempre dispuestos a ofrecer al electorado más de lo que el Gobierno hace (outbidding), convencidos de que no serán llamados a responder por sus exageradas promesas. Su oferta irá mucho más allá de políticas públicas concretas y diferenciadas. Se referirá a las reglas de juego, es decir, la Constitución, el sistema político-económico y, llegado el caso, también a las alianzas internacionales. En el pluralismo polarizado, a izquierda y derecha se encontrarán partidos antisistema que, según Sartori, tienen como objetivo cambiar el sistema, las reglas del juego político y económico (una vez el capitalismo, hoy la globalización). El objetivo propuesto, el cambio perseguido es, por tanto, el hundimiento del sistema. Frente a un objetivo de estas características, declarado más o menos abiertamente, los partidos que defienden el sistema, así como sus electores, no pueden permitir la alternancia. Por lo tanto, en los sistemas caracterizados por un pluralismo polarizado la alternancia en el Gobierno es imposible, resulta impracticable. Aún más, ni siquiera es deseada por los propios partidos antisistema, los cuales prefieren presentarse no para una alternancia reversible, sino para una alternancia irreversible.

Desde 1946 a 1992 Italia no registró ni la una, la alternancia en el Gobierno entre coaliciones, ni la otra, a saber, la sustitución de la coalición de Gobierno por una coalición capaz de cambiar incluso el sistema. Resulta más que razonable sostener que esta ausencia de alternancia tuvo consecuencias políticas muy negativas para la DC y el PCI. Como las tuvo, en general, para el sistema de partidos in toto. En el curso del tiempo, los dos mayores 
partidos sobre los que descansaba el sistema se fueron erosionando sin haber sabido proceder a un recambio fisiológico de sus élites. Los demás partidos con excepción de la tentativa, no del todo adecuada, del PSI de producir algún cambio sistémico-, apenas representaban ya a una sociedad que se había transformado profundamente - ya sea económica como social y culturalmente- y en la que los síntomas de la decadencia ya eran visibles ${ }^{3}$.

Las razones del hundimiento del sistema de partidos, como de los mismos partidos, son muchas y han sido estudiadas desde diversas perspectivas ${ }^{4}$. Se podría sostener, en todo caso, que en 1992 todo el sistema de partidos se había agotado sustancialmente. Tanto en lo que toca al formato, algunos partidos estaban en vías de desaparecer, como en lo referido a la mecánica, pues la transformación del PCI contribuyó a reducir el grado de polarización del sistema. Lo que interesa a nuestro objetivo, en concreto, es comprender la aparición de una nueva situación, explicar la dinámica, valorarla y prever las consecuencias del sistema de partidos de 2008 hasta hoy. Esta operación requiere una reflexión, siquiera mínima, sobre el sistema de partidos que hace su difícil aparición en 1994. Desde un punto de vista restringido, pero significativo, es una nueva ley electoral, que lleva el nombre de su ponente, Sergio Mattarella, la que redefine y delimita la modalidad de la competición política, obligando a los partidos a determinados comportamientos de colaboración-competición, premiando las coaliciones electorales o castigándolas e infrarrepresentando o, aún más, excluyendo, a los partidos que compiten solos. Sobre la ley electoral y sus características recomiendo algunos estudios útiles (Katz, 2001; D'Alimonte, 2001) para concentrar mi atención en la descripción del formato y de la mecánica del nuevo sistema utilizando los criterios recomendados por Sartori.

\section{HUNDIMIENTO, RECUPERACIÓN Y RECAÍDA: 1994-2008}

En cuanto a la forma, el sistema de partidos 1994-2008 es seguramente multipartidista, no limitado en referencia al número de partidos, que son

3 De manera rigurosa y muy convincente, Capussela (2019) deriva la decadencia del sistema de las elecciones, como de las no elecciones, de los propios partidos y de sus consecuencias. Las propuestas de cambio del sistema formuladas por Craxi son expuestas y analizadas en el volumen editado por Acquaviva y Covatta (2010). Los resultados fueron absolutamente marginales.

4 Gilbert (1995) las presenta y analiza de manera sintética, correcta y precisa. 
regularmente más de cinco o seis, y significativamente menos polarizado 5 . A saber, básicamente sin partidos antisistema, por mucho que, si bien de manera poco realista y oportunista, Rifondazione Comunista intentó mantener esta característica. En esta fase los cambios más profundos vinieron dados por la competición entre los partidos. Conscientes de arriesgarse a ser excluidos de la política, del Parlamento y de los cargos, tanto los neofascistas como los neocomunistas aceptaron la idea y la práctica de una competición centrípeta, evitando extremar sus posiciones. De modo que la predisposición de ambos partidos a formar parte de coaliciones que presentaban su candidatura para gobernar el país llevó el sistema a una competición sustancialmente bipolar ${ }^{6}$.

De este modo se pusieron las premisas y las bases para la alternancia. Si bien de manera no exactamente lineal ni ordenada. Excluyendo las elecciones de 1994, en las que ninguno de los contendientes representaba ni al Gobierno saliente ni a la oposición de aquel Gobierno, en las dos elecciones sucesivas, las de 1996 y 2001, el vencedor no había estado en el Gobierno anteriormente. Sin embargo, resulta más complicado definir quién había constituido la oposición, por lo que todo el análisis sobre la alternancia en el sistema de partidos 1994-2001 está destinado a permanecer indefinido e incompleto (Valbruzzi, 2011). No resulta sencillo, por tanto, definir este sistema de partidos. Más allá del número de partidos y de la modalidad de la competición, entran en escena nuevos partidos y desaparecen algunos de los viejos. En este sentido, la aparición de Forza Italia en 1994 es digna de atención porque fue el protagonista de la política italiana durante casi veinte años. Su fortaleza electoral y política obligó al centro-izquierda a (re)organizarse de manera distinta, aunque nunca de manera definitiva ni duradera ${ }^{7}$. En todo caso, la

5 Los neofascistas, que perdieron su condición de partido antisistema, en 1994 fueron incluidos de inmediato en el Polo del Buon Governo, la coalición hábilmente construida por Silvio Berlusconi. Tras cambiar nombre y símbolo en enero de 1991, el PCI se convirtió en el Partito Democratico della Sinistra (PDS) e intentó construir una coalición reformista para la ocasión, llamada Progressisti, con Verdi y Rifondazione Comunista. Sin embargo, la operación funcionó poco y mal. A pesar de que los ex y postcomunistas intentaron dar vida a un polo alternativo al organizado por Berlusconi, la competición solamente se convirtió en bipolar cuando se unieron a estos los ex y postdemocristianos.

6 En 1996 la Lega Nord no participó en la competición bipolar, condenando a la derrota a la coalición de Berlusconi. Una operación similar fue llevada a cabo por Rifondazione Comunista en 2001, contribuyendo de manera importante al pésimo resultado electoral del centro-izquierda.

7 Chiaramonte y Emanuele (2017) ofrecen sugerencias útiles para entender la desaparición de los viejos partidos, la aparición de nuevos partidos y, en general, los 
fusión que llevó al nacimiento del Partido Democratico en 2007 alimentó las expectativas de aquellos que esperaban una profunda reestructuración del sistema de partidos y una competición bipolar limpia.

Para que la consolidación de un sistema de partidos se produzca de manera efectiva, hace falta no solo que su dinámica — léase, su modalidad de competición - sea ampliamente aceptada, respetada y se mantenga invariable en el tiempo, logrando traducirse en prácticas cuyos resultados no sean puestos en discusión continuamente. Necesita, a su vez, que los actores sean estables, duraderos, si no los mismos. Pocos de estos elementos, sin embargo, se han dado cita en el sistema de partidos italiano entre 1994 y 2008. Los abordaré en secciones posteriores de este artículo. Los estudiosos de la materia han llegado a dos conclusiones que parecen convincentes. La primera es que, sin duda, al menos en las fases iniciales, este sistema de partidos se caracterizó como pluralismo despolarizado (Sani, 1995). La segunda es que la despolarización fue acompañada por una progresiva desestructuración (Pappalardo, 2001). Por lo tanto, durante el periodo 1994-2008 el sistema de partidos italiano nunca consiguió consolidarse. Las pruebas son numerosas; me remito, en particular, a las que tocan a los propios partidos. En modo especial, al mundo del centro-izquierda y de sus líderes. En el centro-derecha los tres principales líderes siguieron siendo Silvio Berlusconi (Forza Italia), Gianfranco Fini (Alleanza Nazionale) y Umberto Bossi (Lega). También el pequeño partido de los exdemocristianos vivió el liderazgo sin oposición de Pierferdinando Casini ${ }^{8}$. En el centro-izquierda, sin embargo, se registraron distintas formas tanto de diversificación-fragmentación de las propias organizaciones como de inestabilidad y sustitución de liderazgos.

La agregación llamada Ulivo, analizada a fondo recientemente por Olasio (2018), tuvo un solo líder, Romano Prodi. El cual, en realidad, nunca quiso ni asumir el rol ni desempeñar las funciones propias de capo politico del Ulivo, circunscribiendo su acción al rol más específico y restringido de jefe del Gobierno9. Se trató de un error que repitió, de forma agravada, en el periodo 2006-2008. Finalizada de forma abrupta la experiencia de Gobierno del

cambios en los partidos.

8 La larga trayectoria política de Silvio Berlusconi y el impacto de Forza Italia sobre el sistema de partidos en Italia han sido tratados en profundidad por Newell (2019), cuya valoración, por otra parte, considero excesivamente crítica y severa.

9 La experiencia de Prodi como Ministro de Industria (diciembre 1978-marzo 1979) en el Gobierno presidido por Giulio Andreotti, así como el conocimiento de las vicisitudes democristianas, deberían haberle enseńado que, antes o después, todos los líderes del partido mayor de la coalición desafían al presidente del Consejo de Ministros. Sobre todo cuando el líder del partido no posee poder político propio. 
Ulivo, el centro-izquierda tuvo otros dos jefes de gobierno: Massimo D’Alema (octubre 1998-abril 2000) y Giuliano Amato (mayo 2000-marzo 2001). El mayor partido, el PDS, cambió su nombre en 1998 para convertirse en Democratici di Sinistra (DS) y tuvo tres secretarios: D'Alema, Walter Veltroni y Piero Fassino. Fueron, por el contrario, tanto Fausto Bertinotti, secretario de Rifondazione Comunista desde 1994 a 2006, como Antonio di Pietro, fundador y líder de Italia dei Valori desde 1998 a 2014. Otro partido personal como tanto otros. En este mismo sentido, no fue partido personal en menor grado La Margherita, dirigida por Francesco Rutelli desde 2002 hasta su fusión con DS en 2007. En todo este tiempo Silvio Berlusconi puso en marcha, del 2001 al 2007, la coalición Casa delle Libertà, agregando Alleanza Nazionale, la Lega Nord y el Centro Cristiano Democratico (CCD), definido después como Unione dei Democratici Cristiani e di Centro (UDC). Después, tras la victoria en las elecciones de 2008, nació el Popolo della Libertà como fruto de la fusión de Forza Italia y Alleanza Nazionale, aunque el PdL saltaría por los aires en julio de 2010. Este impulso de agregación del centro-derecha era una respuesta, en buena medida, al fenómeno que caracterizó la década: la formación del Partito Democratico. Precedido por los congresos de disolución de DS y La Margherita en la primavera de 2007 y oficializado con la elección del secretario Walter Veltroni en octubre de 2007.

\section{2007: UN NUEVO PARTIDO, NO UN NUEVO SISTEMA DE PARTIDOS}

El acontecimiento del año 2007, el nacimiento del Partito Democratico, ciertamente muy importante, solo es parcialmente asimilable al fenómeno de 1994, es decir, a la irrupción de Forza Italia en la escena política italiana. El PD nace como fusión - «fusión fría» como la definieron sus críticos, es decir, sin entusiasmo ni movilización por parte de sus partidarios- de dos partidos que ya habían agotado su largo recorrido. Y que se dirigían, además, primero hacia la ruinosa caída del segundo Gobierno guiado por Romano Prodi, registrada en enero de 2008, y segundo hacia la que sería una durísima derrota electoral en abril de ese mismo año 2008. El PD no era y no podía ser, por la modalidad de su formación (para cuya profundización remito a Pasquino, 2009), más que un partido nuevo implantado en el tronco de dos partidos ya existentes. Con una vieja clase política que se mudó, con todas sus armas y equipaje, a los puestos de importancia y liderazgo de la neonata organización. Por el contrario, en 1994 Berlusconi dio vida a un nuevo partido que, incluso agregando trozos de partidos de centro en ruina, se fundó, en buena medida, sobre profesionales que provenían de la sociedad (civil), como el propio 
Berlusconi. El efecto combinado del nacimiento de Forza Italia, que se propuso unir en coalición todo el centro-derecha, con la nueva ley electoral de Mattarella, que incentivaba de manera decisiva la presentación de una candidatura única en cada uno de los colegios uninominales, condujo, incluso determinó, la aparición de una democracia sustancialmente bipolar, mayoritaria y susceptible de alternancia.

Resulta interesante subrayar cómo los ideólogos del Partito Democratico recuperaron prácticamente las mismas palabras clave bajo la bandera de la democracia de la alternancia ${ }^{10}$. Y, sobre todo, cómo el secretario Veltroni configuró tanto su acción política como su campaña electoral exaltando la «vocación mayoritaria» del PD. Sin embargo, la ley electoral había cambiado de manera muy significativa respecto a 1994. Mas conocida como Porcellum y aprobada en 2005, la ley electoral que lleva el nombre del senador leghista Roberto Calderoli, reunía tres características cruciales: un sistema proporcional; un premio de mayoría al partido o a la coalición que obtuviese, incluso, un solo voto más; listas bloqueadas y, con carácter subsidiario, aunque no se trata de un elemento del todo marginal ni irrelevante, la posibilidad de pluricandidaturas, hasta cinco. En estas condiciones, el bipolarismo podría haberse afirmado exclusivamente si, en efecto, se hubiesen constituido dos coaliciones. Por el contrario, la vocación mayoritaria del partido de Veltroni, que sin embargo estableció una alianza con Italia dei Valori de Di Pietro, estaba destinada a ser un gesto vacío. Hubiera sido, de todos modos, un bipolarismo electoral una tantum, con partidos que se aliaban por conveniencia, nunca por convicción.

10 Utilizo de manera muy poco apropiada la expresión «ideólogos del PD». De hecho, las dos características ideológicas del partido fueron establecidas no en un manifiesto político-programático, sino, de un lado, en el hecho de confiar la elección de las candidaturas a los cargos electivos a elecciones primarias, y de otro, en la resolución estatutaria que hacía coincidir el papel de secretario del partido con el de jefe del Gobierno (abandonada con una pequeña reforma en noviembre de 2019). Para una poderosa y eficaz crítica del Estatuto del PD se recomienda acudir a Floridia (2019). Consúltese también el artículo de L. Fasano (2020). Resulta del todo evidente que el PD había abandonado, básicamente, cualquier anclaje ideológico en un tiempo caracterizado por el final de las ideologías para todos los partidos, no solo los italianos. Sin embargo, que ese fenómeno fuese acompañado de la desaparición de las culturas políticas no era, en absoluto, inevitable (Pasquino, 2015). 


\section{LA DESESTRUCTURACIÓN DEL SISTEMA DE PARTIDOS}

Fotografiado después de las elecciones de abril de 2008, el sistema de partidos italiano merece ser definido como multipartidismo moderado - con solo seis partidos representados en el Parlamento-, de dinámica centrípeta, despolarizado — sin ningún partido antisistema—. Sin embargo, haciendo uso de la terminología de Pappalardo (2001), se trata de un sistema todo lo contrario a consolidado: más bien, «desestructurado». Resulta posible y útil distinguir dos componentes del fenómeno de la desestructuración. En primer lugar, se considera que un sistema entra en fase de desestructuración cuando el modo en que se produce la interacción y competición entre los distintos partidos cambia de manera repetida y frecuente, cuando no es aceptada por algunos de los partidos en liza y no responde a reglas conocidas y compartidas. Al contrario, estas son discutidas y manifiestan irregularidad. En segundo lugar, la desestructuración también hace referencia a uno o varios partidos del sistema. Se refiere a su aparición o desaparición, debilitamiento electoral, aumento de votos y/o escaños y (des)organización interna, en referencia a la división en corrientes y a los cambios frecuentes e imprevistos de liderazgo.

La componente de la desestructuración que toca a la modalidad de la interacción y competición está condicionada esencialmente, como puede intuirse con facilidad, por las leyes electorales y su naturaleza, dependiendo de si son mayoritarias o proporcionales o contienen otras clausulas específicas. Por ejemplo, de 2008 a 2018 Italia ha pasado de arbitrar su competición política con una ley electoral proporcional con listas bloqueadas y premio a la mayoría (ley Calderoli, conocida como Porcellum) a una ley electoral, impulsada por el diputado del Partido Democratico Ettore Rosato (ley Rosato), dos tercios proporcional, un tercio mayoritario en colegios uninominales y un umbral del $3 \%$. Además, vale la pena recordar que ambas leyes prevén pluricandidaturas que permiten a los dirigentes de los partidos tanto asegurarse su propia elección como nominar candidatos que quieren convertir en electos. Finalmente, ambas leyes han sido elaboradas teniendo como objetivo el poder de los partidos y de sus dirigentes, no el de los electores. A los cuales, de hecho, solo les es concedido trazar una cruz sobre el símbolo del partido o, en el caso de la ley Rosato, sobre el nombre del candidato ${ }^{11}$.

11 En lo tocante a la ley Rosato (y a su predecesora Italicum, declarada parcialmente inconstitucional por la Corte Constitucional) resulta sencillo hacer notar que entre los objetivos latentes está/estaba hacer difícil la vida al M5S, cuya opción política de no coaligarse con otros partidos le colocaba en posición de desventaja en los colegios uninominales, en los cuales, obviamente, el candidato respaldado por una coalición 
Como prueba evidente de que el sistema de partidos no estaba de hecho consolidado, pueden señalarse dos sucesos significativos. En julio de 2010 Alleanza Nazionale abandonó el Popolo della Libertà, mientras que Italia dei Valori había dejado ya su alianza con el Partito Democratico, formando un grupo parlamentario autónomo tanto en la Cámara de los Diputados como en el Senado. Entre 2008 y 2013 también se produjeron otros tres acontecimientos de notable relevancia que apuntan en la misma dirección. El primero fue la dimisión de Walter Veltroni como secretario del Partito Democratico en febrero de 2009, quien sería sustituido por Pierluigi Bersani después de una breve fase de regencia del vicesecretario Dario Franceschini. El segundo suceso importante fue la crisis del cuarto Gobierno Berlusconi, quien, obligado a dimitir en noviembre de 2011, fue sustituido por un Gobierno no político liderado por el profesor Mario Monti en el periodo 2011-2013 (Pasquino y Valbruzzi, 2015: capítulo 3). El tercero fue la potente irrupción del M5S en las elecciones locales, caracterizada por victorias en ayuntamientos del sur y en el norte de Italia que decantaron, finalmente, la decisión del M5S de participar en las elecciones de febrero de 2013. Por añadidura, el presidente del Consejo de Ministros, Mario Monti, también decidió participar en las elecciones de 2013 con su propia lista electoral, Scelta Civica, provocando gran irritación y decepción en el presidente de la República, Giorgio Napolitano. En su logo se inscribían las palabras "Con Monti per l'Italia», contribuyendo así a crear al enésimo vehículo electoral personal. A pesar de que el resultado de Scelta Civica no confirmó las ambiciosas expectativas creadas en torno al nuevo partido de Monti, su ingreso en el Parlamento era la señal de que el sistema de partidos era de todo menos un sistema consolidado ${ }^{12}$.

En cierto sentido, el indicador más convincente de la citada ausencia de estructuración del sistema de partidos italiano lo refleja su índice de volatilidad electoral. Es decir, el porcentaje de los electores que cambia su comportamiento de unas elecciones a otras. Los datos son absolutamente reveladores (véase también los datos ofrecidos por Chiaramonte y Emanuele, 2017: 266).

gozaba de una mayor posibilidad de éxito. Sin embargo, los hechos han mostrado que las desventajas del M5S han sido bastante limitadas.

12 Entre otras cosas, el repetido ingreso en el Parlamento de nuevos partidos señala que los partidos existentes no pueden erigirse en partido-cártel para proteger su posición. Lo que parece desmentir, parcialmente, la tesis formulada por Katz y Mair (1995), y reiterada, con muy pequeños ajustes (2009). 
Gráfico 1. La volatilidad electoral en Italia 1948-2018

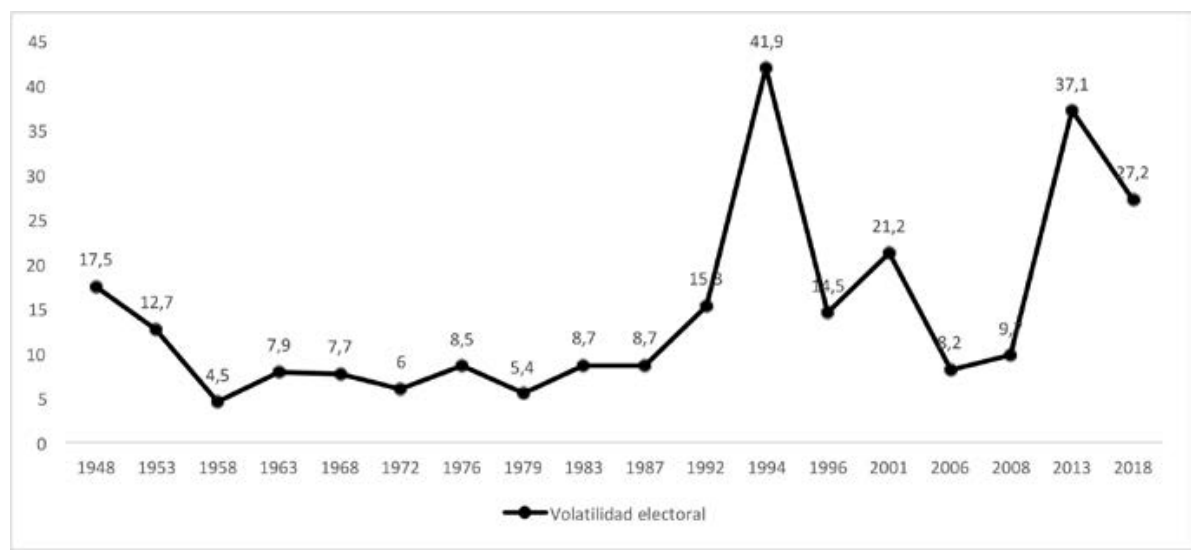

Fuente: Valbruzzi (2018: 181) a partir de la elaboración del Istituto Cattaneo.

En el periodo 1994-2008 la elección más volátil fue la celebrada en 1994, caracterizada por la entrada en escena de un nuevo partido, Forza Italia. En el periodo 2013-2018 no solo se registra la aparición de un nuevo protagonista político, el M5S en 2013, sino que debe considerarse también el gran cambio electoral que suponen los comicios de 2018. Un cambio imputable a la notable insatisfacción del electorado italiano y su búsqueda de alternativas posibles. Los viejos partidos, Forza Italia y el Partito Democratico, resultaron significativamente redimensionados. Scelta Civica desaparece. El Movimento Cinque Stelle consigue un porcentaje de votos equiparable a los registrados por la DC y comparable al máximo electoral del PCI en 1976. A partir de su cambio de líder, pasando del viejo fundador del partido Umberto Bossi al joven y combativo Matteo Salvini, la Lega consigue acreditarse como un partido significativamente renovado, consiguiendo cuadruplicar su resultado y ganando más votos, en términos absolutos, que el M5S. Solo para completar la información, introduzco el dato decepcionante de Liberi e Uguali (LeU), sustancialmente compuesta por aquellos que fueron obligados a salir del Partito Democratico ${ }^{13}$.

13 Liberi e Uguali (LeU) fue un grupo parlamentario constituido en enero de 2017 por un número de parlamentarios, más bien pequeńo, que abandonaron el Partito Democratico guiado por Matteo Renzi y se presentaron, después, a las elecciones de 2018. 
Tabla 1. Variación de votos y liderazgos en el sistema de partidos italiano 2008-2018

\begin{tabular}{lccccc}
\hline & $\mathbf{2 0 0 8}$ & $\mathbf{2 0 1 3}$ & $\mathbf{2 0 1 8}$ & $\mathbf{2 0 0 8 - 2 0 1 8}$ & $\begin{array}{c}\text { Numero } \\
\text { di leader }\end{array}$ \\
\hline Forza Italia & $13.629 .464^{*}$ & 7.332 .134 & 4.596 .956 & -4.325 .382 & 1 \\
\hline $\begin{array}{l}\text { Partito } \\
\text { Democratico }\end{array}$ & 12.095 .306 & 8.646 .034 & 6.161 .896 & -5.933 .409 & 7 \\
\hline Lega (Nord) & 3.024 .543 & 1.390 .534 & 5.698 .687 & +2.674 .144 & 2 \\
\hline Fratelli d'Italia & & 666.765 & 1.499 .550 & +832.785 & 1 \\
\hline $\begin{array}{l}\text { Movimento 5 } \\
\text { Stelle }\end{array}$ & & 8.691 .406 & 10.732 .066 & +2.040 .660 & 2 \\
\hline Scelta Civica & & 2.823 .842 & & & 1 \\
\hline LeU & & 1.114 .298 & & 1 \\
\hline
\end{tabular}

* Este resultado se refiere a los votos obtenidos por el Popolo della Libertà, lista unitaria formada por Forza Italia y Alleanza Nazionales. Si restamos los votos de AN en las elecciones de 2006, podemos atribuir a Forza Italia cerca de 8.922 .338 votos. ${ }^{* *}$ Por orden, los líderes son: Forza Italia (Berlusconi); Partito Democratico (Veltroni, Franceschini, Bersani, Epifani, Renzi, Martina, Zingaretti); Lega (Bossi, Salvini); Fratelli d'Italia (Meloni); Movimento Cinque Stelle (Grillo, Di Maio).

Fuente: elaboración propia.

Tabla 2. Escaños en la Cámara de Diputados y el Senado de los tres principales partidos después de las elecciones de 2018*

\begin{tabular}{lccc}
\hline & Camera & Senato & Totale \\
\hline Movimento 5 Stelle & 227 & 111 & 338 \\
\hline Partito Democratico & $112^{* *}$ & $52^{* * *}$ & 164 \\
\hline Lega & 58 & 125 & 183 \\
\hline
\end{tabular}

* Se advierte que debido a los cambios que se han registrado en la actual legislatura, muchos de ellos en marcha, estos números no corresponden exactamente con los de las actuales fuerzas parlamentarias. En particular, el M5S ha sufrido algunas deserciones ( 9 diputados y 7 senadores) y el PD tras la escisión de Italia Viva ha perdido 17 senadores y 28 diputados. ** Más 14 escaños de LeU. *** Más 4 escaños de LeU.

Fuente: elaboración propia. 
La teoría de las coaliciones ofrece indicaciones sobre los modos en los que se forman Gobiernos en los contextos multipartidistas. En el caso italiano, dado que el Gobierno debe conseguir un voto de confianza en el Parlamento, las coaliciones de Gobierno nacen y (sobre)viven cuando son sostenidas por una mayoría absoluta de parlamentarios. Por lo tanto, resulta fácil deducir que la distribución de los escaños en la Cámara y el Senado solo permitía la formación de dos coaliciones ganadoras. En orden, Movimento Cinque Stelle-Lega (352 escańos en la Cámara de los Diputados y 169 en el Senado), o Movimento Cinque Stelle + Partito Democratico (339/163). Sabemos también que, por convención, la responsabilidad de iniciar las negociaciones para la formación de Gobierno se confía, inicialmente, al líder del partido que ha obtenido más votos y que dispone de más escaños. En este caso, por tanto, el pentastellato Luigi Di Maio.

El secretario del PD, Matteo Renzi, decidió llevar el partido a la oposición sin convocar, siquiera, a los órganos del partido: Secretaría y Dirección. En cierta manera, como de otra parte reivindicaron los dos protagonistas, Di Maio y Salvini, la coalición M5S-Lega estaba compuesta por los vencedores de las elecciones. Sin embargo, ni siquiera esta coalición puede entenderse como un caso de alternancia. En efecto, a pesar de los numerosos cambios de Gobierno registrados entre 2001 y 2018, no es posible definirlos como casos de alternancia. De hecho, en este periodo no encontraremos nunca una coalición de Gobierno que haya sido derrotada por la oposición existente en el curso de la legislatura, pues en ninguno de los casos se registra la existencia de una oposición, claramente delineada y organizada, que consiguiese vencer a un Gobierno creado en referencia a los votos obtenidos en las elecciones precedentes ${ }^{14}$. Tanto en 2013 como en 2018, los Gobiernos se han formado no sobre la base de la oferta política realizada al electorado, sino, como tantas veces sucede en las democracias parlamentarias, aunque no siempre, en función de los votos y los escaños obtenidos, así como las opciones numérica y políticamente realizables.

En coherencia con mis afirmaciones, todas las cuales derivan de una doble fuente de conocimiento - la teoría de las coaliciones y la experiencia de

14 En 2001 el Gobierno de centro-izquierda derrotado no fue el del Ulivo, que había vencido en las elecciones de 1996, sino el Gobierno de centro-izquierda que toma el relevo de Prodi y se apoyó decisivamente en sesenta parlamentarios tránsfugas que provenían del centro-derecha. A su vez, la oposición que obtuvo la victoria en 2001 no estaba compuesta por los partidos que habían perdido en 1996, dado que la Lega no había formado parte de la coalición. Precisamente, su reincorporación a la coalición de centro-derecha, llamada Casa delle Libertà, permite y produce la victoria. 
la formación de Gobiernos italianos (Pasquino, 2020) — me atreveré a afirmar que el resultado de la crisis relámpago de agosto de 2019, desatada por la arrogancia política e ignorancia constitucional de Matteo Salvini, era casi inevitable ${ }^{15}$. En el Parlamento, como hemos visto, era posible formar otra coalición, M5S-PD, que podía disfrutar de la mayoría absoluta de los escaños, tanto en la Cámara como en el Senado. Se trataba del Gobierno alternativo que, de hecho, vio rápidamente la luz en septiembre de 2019: el llamado Gobierno Conte II, sostenido también por los parlamentarios de LeU. En el horizonte sigue el peligro de que entre los dos, M5S y PD, quieren posponer y evitar una coalición de la derecha -Lega, Forza Italia y Fratelli d'Italia- guiada por Salvini, a la que los sondeos continúan señalando como opción mayoritaria del electorado y destinada a una notable victoria.

\section{BALANCE Y VALORACIÓN FINAL}

Reflexionando sobre los tres sistemas de partidos presentados en secuencia histórica por Valbruzzi (2013), me parece oportuno subrayar que el sistema nacido de las elecciones de 2013 ha encontrado su reafirmación y continuación después de las elecciones de 2018. Desde entonces, el formato del sistema de partidos italiano se ha definido incluso con mayor claridad, en referencia a los criterios de Sartori, come sistema multipartidista con tendencia al aumento de partidos y debilitamiento de los dos partidos, M5S y PD, que forman actualmente el Gobierno. De modo que el sistema actual se compone de seis partidos relevantes: la Lega, el Movimento Cinque Stelle, Partito Democratico, Forza Italia, Fratelli d'Italia e Italia Viva. En el último caso, se trata de un partido personalista, largamente esperado y creado en septiembre de 2019 por el exsecretario del PD, Matteo Renzi ${ }^{16}$.

Renzi dispone de cuarenta diputados y quince senadores, y su consenso en los sondeos no va más allá del 3-4\%. Renzi ha conseguido algún ministro, viceministro y subsecretario en el Gobierno Conte II. Sus números actuales le

15 Arrogancia política, dado que Salvini dijo explícitamente querer «plenos poderes» (expresión que se remonta a Benito Mussolini); ignorancia constitucional, en tanto que Salvini parecía no saber que el poder de disolver el Parlamento recae, completamente, en las manos del presidente de la República, el cual también tiene el poder de nombrar un nuevo Gobierno.

16 Extraño y deplorable caso de un exsecretario de partido que opera, a sangre fría, para, en primer lugar, empujar fuera de su partido a aquellos que disentían de su línea política (después convertidos en LeU) y, en segundo lugar, para crear una escisión convirtiéndose en competidor de su partido original. 
permiten explotar desprejuiciadamente su poder de chantaje, es decir, de amenazar con hacer caer al Gobierno. En términos reales, una crisis de Gobierno, que probablemente conllevaría nuevas elecciones, no conviene a Renzi en tanto que la ley electoral pone en franca desventaja a los partidos pequeños. Sabemos lo mucho que las leyes electorales determinan la forma de un sistema de partidos, permitiendo la aparición de nuevos y pequeños partidos, pero haciendo su vida difícil y la supervivencia complicada. Este fenómeno, al menos en sus rasgos principales, no escapa a Renzi y sus colaboradores. Autores de una ley electoral bautizada como Italicum, desmantelada por la Corte Constitucional, y después refugiados en la ley Rosato, que lleva el nombre del jefe del grupo parlamentario del PD en la Cámara de los Diputados y, actualmente, coordinador y reclutador de Italia Viva.

En cuanto a la dinámica del sistema de partidos, el M5S ha entrado en la lógica de funcionamiento de la democracia parlamentaria y, por tanto, se ha visto obligado a abandonar su carga antisistema ${ }^{17}$. Por tanto, podemos hablar, en un cierto sentido, de un multipartidismo moderado, aunque siga siendo importante el grado de polarización perceptible, tanto como la distancia política e institucional que separa a Forza Italia y Fratelli d'Italia del Movimento Cinque Stelle ${ }^{18}$. Frecuentemente, incluso en las situaciones en las que los partidos que se registran son cinco o seis, se puede afirmar que una dinámica de competición política y electoral es de tipo bipolar. Sin embargo, puesto que el M5S continuará presentándose como un polo autónomo, y el $\mathrm{PD}$ tampoco renunciará a esa caracterización, es probable que en el futuro más cercano el sistema de partidos italiano tenga una dinámica tripolar ${ }^{19}$. De hecho, a pesar de la relativa homogeneidad de los tres partidos que constituyen el centro-derecha, la Lega puede, siempre que lo quiera, dar o negar cohesión a los tres partidos. Sin embargo, resulta improbable que el partido de Salvini desee volver a un acuerdo con el M5S, para el cual tampoco contarían con los números necesarios ${ }^{20}$.

17 Sin embargo, las pulsiones antiparlamentarias están lejos de haber desaparecido en Italia, como ha mostrado la drástica reducción del número de parlamentarios. De 630 a 400 diputados, y de 315 a 200 senadores.

18 Resulta más complejo el discurso sobre la distancia entre la Lega y el M5S, que no ha impedido, en todo caso, su común experiencia de Gobierno entre junio de 2018 y agosto de 2019, aunque esta va acentuándose con el retorno de la Lega a la oposición.

19 Un dirigente autorizado del PD, Dario Franceschini, actualmente ministro de Bienes Culturales, ha sostenido, repetidamente, que la alianza entre el PD y el M5S debe continuar en el futuro y estructurarse como un verdadero y auténtico polo. Oficialmente, en esta fase, se trata de una propuesta minoritaria.

20 Sin embargo, Salvini ha alimentado la ambición de Di Maio más de una vez prometiéndole el cargo de presidente del Consejo de Ministros si se volviese a una 
Prácticamente despolarizado a todos los efectos, en ningún caso el sistema de partidos italiano puede considerarse estructurado. Es decir, en condiciones de mantenerse en el formato salido de las elecciones de 2018. Forza Italia continua su lento declinar. Debido, de un lado, a la erosión de votos y pérdida de personal político, tanto a favor de la Lega como de Fratelli d'Italia; del otro, a la sustancial ausencia de una propuesta política clara. Berlusconi, cuyo liderazgo resulta evanescente a estas alturas, parece tentado por la idea de construir un nuevo órgano político. En lo tocante al centroizquierda, el otro protagonista de la década, el Partito Democratico, se muestra desgastado y lento a la hora de dar respuesta a los desafíos; incapaz, en definitiva, de una elaboración estratégica y, finamente, sin aliados ${ }^{21}$. Puede esperar, como mucho y gracias a la decadencia electoral del M5S, un reequilibrio numérico con el Movimento, o quizás un sorpasso, pero cuesta abajo.

Dos elementos, imprevisibles en sus dimensiones, parecen estar en disposición de producir algún cambio ulterior, aunque probablemente no decisivo. De un lado, la volatilidad electoral, mucho más que la participación electoral, cuyo declinar es lento y débil, podría premiar a algún nuevo sujeto político, de momento no visible. Del otro, el verdadero motor del próximo cambio será la trayectoria del Movimento Cinque Stelle: su recuperación, en estos momentos complicada, o su posible declive, acelerado y acentuado, liberaría algunos millones de electores, los cuales buscarían una opción distinta para dar expresión a su falta de satisfacción con la política italiana, los partidos existentes y el funcionamiento general de las instituciones. Aunque, como sabemos gracias a los estudios electorales, muchos de estos electores insatisfechos, aproximadamente uno de cada tres, volverían o se refugiarían en la abstención ${ }^{22}$.

Quien conoce un solo país, decía Giovanni Sartori, no conoce ni siquiera ese país. Se trataba de una invitación a reflexionar sobre las causas de la transformación de los sistemas de partidos italiano teniendo en cuenta los desarrollos de otros países, por ejemplo como España y, al menos en parte, Alemania. Utilizo el plural «sistemas de partidos» toda vez que, como ya he señalado

fórmula de gobierno Lega-M5S.

21 Para más y mejor sobre el PD, véase Fasano (2020).

22 En el momento en el que escribo este artículo, en muchas plazas de Italia, empezando por la de Bolonia, se registran manifestaciones de varios miles de personas, principalmente, aunque no solo, jóvenes que protestan con la Lega, contra Salvini y sus políticas y contra la difusa aparición de comportamientos neofascistas. Este nuevo movimiento, autodefinido como las Sardinas, también intenta empujar hacia acciones más decisivas e incisivas a la izquierda, es decir, al PD. Es muy probable que entre estos jóvenes haya electores pasados y presentes del M5S. Resulta, con todo, difícil predecir el futuro. 
varias veces con anterioridad, creo que es posible, útil y correcto identificar tres sistemas de partidos, periodizándolos como sigue: 1946-1992, pluralismo polarizado; 1994-2013, pluralismo despolarizado competitivo, y 2013-, tripolarismo desestructurado.

El sistema de partidos que emerge después de las elecciones de 2018 tiene unas características suficientemente marcadas que considero útil subrayar de nuevo. En cuanto al formato, como se ha señalado, se trata de un multipartidismo moderado, con cinco o seis partidos que cuentan todos con poder o potencial de gobierno y de chantaje, pero ninguno abiertamente antisistema ${ }^{23}$. En cuanto a la dinámica: de un lado, la competición es tripolar y fundamentalmente centrípeta. Pero aquello que me parece más importante es que nacen y mueren partidos relevantes y, sobre todo, permanece en cifras elevadas, cerca del $30 \%$, la cantidad de electores que están insatisfechos con la oferta política. Electores que, por tanto, están dispuestos, como así lo hacen, a cambiar su comportamiento electoral de una a otra elección.

Como puede observarse en el gráfico sobre la volatilidad en Italia en perspectiva histórica, a la volatilidad inicial -si bien de otra parte bastante contenida, que responde al asentamiento del sistema de partidos, en particular a la izquierda, y del aprendizaje de la modalidad de competición- le siguen casi cuarenta años de baja volatilidad, interrumpida por el éxito de la Lega en 1992. La fase sucesiva está caracterizada por dos máximos y medio. El primero, en 1994, es el producto conjunto de la desaparición de los partidos centristas y de la irrupción de Forza Italia en el panorama de partidos italiano. El segundo máximo, apenas inferior al primero, responde a la irrupción electoral del M5S en 2013. En ambos casos, más de un tercio de los electores italianos cambiaron su comportamiento electoral. En este sentido, que el sistema de partidos esté lejos de estar consolidado lo confirma que más del $25 \%$ de los electores cambiaron su voto del 2013 al 2018. Y los sondeos sugieren que siguen en curso movimientos similares.

En Italia, en definitiva, hemos asistido a dos fenómenos no muy diferentes de cuanto ha sucedido en otros países de Europa: primero, el declive de los principales partidos y el nacimiento de nuevos partidos, muchas veces con características populistas; segundo, una remarcable movilidad del electorado sostenida por una nueva oferta electoral que ha encontrado una respuesta positiva por parte del electorado. En el caso italiano, el hundimiento de la

23 Naturalmente, resulta lícito preguntarse si una victoria electoral del centro-derecha italiano, con la primacía de Salvini y Meloni, no constituye un desafío al sistema, léase antisistema, si pretenden, efectivamente, poner en práctica su soberanismo, consistente en la salida del euro y la Unión Europea. 
partitocrazia creó un vacío que parecía haber sido colmado, primero, por Forza Italia de Silvio Berlusconi y, después, por el Partito Democratico. Pero no ha sido así. La aparición del M5S y, después, la expansión acelerada y significativa de la Lega suscitan la pregunta sobre su duración y persistencia. Por último, respecto a otras democracias europeas, Italia manifiesta un ulterior fenómeno problemático, a saber, que resulta difícil de analizar y complicado de afrontar políticamente. Los sistemas de partidos del 1994-2013 y del escenario post-2018 han estado y están notablemente influenciados por las leyes electorales utilizadas. Resulta bastante simple hipotetizar que la reforma de la ley Rosato podrá conducir a una ulterior redefinición del sistema de partidos italiano. También por esto la desestructuración del sistema de partidos italiano parece destinada a persistir, con las muchas e inevitables consecuencias negativas que traería al funcionamiento del sistema político y para la calidad de la democracia italiana (Pasquino, 2020)

\section{BIBLIOGRAFÍA}

Acquaviva, G. y Covatta, L. (coords.) (2010). La "grande riforma» di Craxi. Venezia: Marsilio. Capussela, A. (2019). Declino. Una storia italiana. Roma: Luiss University Press.

Chiaramonte, A. y Emanuele, V. (2017). Party system volatility, regeneration and de-institutionalization in Western Europe (1945-2015). Party Politics. 23 (4), 376-388. Disponible en: https://doi.org/10.1177/1354068815601330.

D'Alimonte, R. (2001). Mixed Electoral Rules, Partisan Realignment, and Party System Change in Italy. En M. S. Shugart y M. P. Wattenberg (coords.). Mixed-Member Electoral Systems (pp. 323-350). Oxford: Oxford University Press. Disponible en: https:// doi.org/10.1093/019925768X.003.0016.

Eckstein, O. (1968). Political parties, Party Systems. International Encyclopedia of the Social Sciences, 11, 436-453.

Fasano, L. M. (2020). El Partito Democratico. Del partido de los reformistas al partido fallido. Un primer balance (2007-2019). Revista de Estudios Politicos, 189, 127-166.

Floridia, A. (2019). Un partito sbagliato. Democrazia e organizzazione nel Partito Democratico. Roma: Castelvecchi.

Galli, G. (1967). Il bipartitismo imperfetto. Comunisti e democristiani in Italia. Bologna: Il Mulino.

Gilbert, M. (1995). The Italian Revolution: The End of Politics, Italian Style. Boulder (Colorado): Westview Press.

Katz, R. S. (2001). Reforming the Italian Electoral Law, 1993. En M. S. Shugart y M. P. Wattenberg (coords.). Mixed-Member Electoral Systems (pp. 96-122). Oxford: Oxford University Press. Disponible en: https://doi.org/10.1093/019925768X.003.0006. 
— y Mair, P. (1995). Changing Models of Party Organization and Party Democracy: The emergence of the cartel part. Party Politics, 1(1), 5-31. Disponible en: https://doi.org/1 $0.1177 / 1354068895001001001$.

Newell, J. L. (2019). Silvio Berlusconi: A Study in Failure. Manchester: Manchester University Press. Disponible en: https://doi.org/10.7765/9781526133946.

Olasio, A. (2018). Il tempo dell'Ulivo. Bologna: Il Mulino.

Pappalardo, A. (2001). Il sistema partitico italiano fra bipolarismo e destrutturazione. Rivista Italiana di Scienza Politica, 31 (3), 561-600.

Pasquino, G. (coord.) (2009). Il Partito Democratico. Elezione del segretario, organizzazione e potere. Bologna: Bononia University Press.

- (2015). La scomparsa delle culture politiche in Italia. Paradoxa, 9 (4), 13-26.

— (2020). Italian Democracy. How It Works. London: Routledge. Disponible en: https:// doi.org/10.4324/9780203732175.

- y Valbruzzi, M. (2015). A Changing Republic. Politics and Democracy in Italy. Novi Ligure: Edizioni Epoké.

Sani, G. (1995). La polarizzazione rivisitata. En G. Pasquino (coord.). La scienza politica di Giovanni Sartori (pp. 153-170). Bologna: Il Mulino.

Sartori, G. (1966). European Political Parties: The Case of Polarized Pluralism. En J. LaPalombara y M. Weiner (coords.). Political Parties and Political Development (pp. 131-176). Princeton: Princeton University Press. Disponible en: https://doi. org/10.1515/9781400875337-006.

- (1976). Parties and party systems. A framework for analysis. Cambridge: Cambridge University Press.

Valbruzzi, M. (2011). Misurare l'alternanza, la sua pratica, la sua mancanza. En G. Pasquino y M. Valbruzzi (coords.). Il potere dell'alternanza. Teorie e ricerche sui cambi di governo (pp. 303-334). Bologna: Bononia University Press.

- (2013). Not a normal country: Italy and its party systems. Studia Politica. Romanian Political Science Review, 13 (4), 617-640.

(2018). Analisi elettorale di un cambiamento radicale. Chi ha vinto e chi ha perso. En M. Valbruzzi y R. Vignati (coords.). Il vicolo cieco. Le elezioni del 4 marzo 2018 (pp. 147-184). Bologna: Il Mulino. 